QUARTERLY OF APPLIED MATHEMATICS

VOLUME LXVIII, NUMBER 3

SEPTEMBER 2010, PAGES 537-556

S 0033-569X(2010)01162-3

Article electronically published on May 27, 2010

\title{
THE DIRICHLET PROBLEM FOR STOKES EQUATIONS OUTSIDE OPEN ARCS IN A HALF-PLANE AND CREEPING FLOW OVER THIN PROFILES
}

\author{
BY \\ P. A. KRUTITSKII \\ KIAM, Miusskaya Sq. 4, Moscow 125047, Russia
}

\begin{abstract}
We study the Dirichlet problem for Stokes equations outside curvilinear open arcs in a half-plane. We prove existence and uniqueness of a classical solution to this problem. We obtain integral representation for a solution in the form of potentials, densities in which can be found as a unique solution of the system of the Fredholm integral equations of the second kind and index zero. The creeping flow of viscous fluid over thin profiles is described by the Dirichlet problem studied in this paper.
\end{abstract}

1. Introduction. The potential theory for the 2-D Stokes equations is constructed in [1] in the case of smooth closed curves and continuous densities in potentials. This theory is applied in [1] to the analysis of solvability of boundary value problems for Stokes equations in interior and exterior simply connected domains bounded by a smooth closed curve. Results obtained in [1] are extended in [2], [3], and [4] to the case of multiply connected domains bounded by smooth closed curves. Boundary value problems in [3], [4] are reduced to the uniquely solvable integral equations. It is known that the problem on the homogeneous viscous flow over an obstacle does not have a physically reasonable solution for Stokes equations in a 2-D case (the so-called Stokes paradox). However, the problem on creeping viscous flow over an obstacle in a half-plane has a solution for Stokes equations [17, 18, 19]. If the fluid fills the upper half-plane, then the boundary of a half-plane can be considered as a bottom.

Potentials for velocities and a potential for pressure for Stokes equations are studied in [7, [8], 9] in the case when the potentials are specified on the open arc while the densities in potentials belong to the weighted Hölder spaces and may have power singularities at the ends of the arc. In [7, [8, 9], the properties of smoothness of potentials are studied, and the limiting formulae for potentials and for their normal and tangential derivatives on the sides of the open arc are obtained. Moreover, the singularities of the pressure potential and singularities of the derivatives of velocity potentials at the ends of the open arc are also studied in [7, [8], [9].

Received November 24, 2008.

2000 Mathematics Subject Classification. Primary 35Q30, 76D07, 31A10, 45F05, 45F15.

(C)2010 Brown University 
In the present paper we study the Dirichlet problem for Stokes equations outside open arcs in a half-plane. The problem models creeping flow of viscous fluid over several thin curvilinear profiles (open arcs). The fluid is placed in the upper half-plane above the plane bottom. We prove existence and uniqueness of a solution. We obtain integral representation for a solution in the form of potentials, densities in which can be found as part of a unique solution of the system of Fredholm integral equations of the second kind and index zero (it is proved that this system is uniquely solvable). In addition, we study singularities of derivatives of velocities at the ends of the open arcs.

The problem of viscous flow over a plate in the form of a half-line has been considered in [16, chapter VI, §6] for non-linear Navier-Stokes equations, and the first asymptotic approximations describing behaviour of velocities at the edge have been obtained. It appears that there are an infinite number of such approximations, but they all satisfy linear Stokes equations. On the other hand, the linear problem being well-posed is uniquely solvable. Therefore, it is interesting to solve the linear overflow problem, to find asymptotics of derivatives of velocities at the edge, and to clarify which asymptotic among the infinite number presented in [16] really takes place. The answer to this question is contained in the present paper as well.

2. Formulation of the problem. By a simple open arc we mean a nonclosed smooth curve of finite length without self-intersections [10. We introduce the Cartesian coordinates $x=\left(x_{1}, x_{2}\right) \in R^{2}$ in a plane. Consider the simple open $\operatorname{arcs} \Gamma_{1}, \ldots, \Gamma_{N}$ of class $C^{2, \lambda}, \quad \lambda \in(0,1]$ in the upper half-plane $R_{+}^{2}=\left\{x: x_{2}>0\right\}$, so that the arcs do not have common points (in particular, endpoints). Denote $\Gamma=\bigcup_{n=1}^{N} \Gamma_{n}$. We assume that the minimum distance between $\Gamma$ and the line $x_{2}=0$ is equal to $\epsilon>0$. Let contour $\Gamma$ be parametrized by the arc length $s$ :

$$
\Gamma_{n}=\left\{x: x=x(s)=\left(x_{1}(s), x_{2}(s)\right), s \in\left[a_{n}, b_{n}\right]\right\}, \quad n=1, \ldots, N
$$

in such a way that $a_{1}<b_{1}<a_{2}<b_{2}<\ldots<a_{N}<b_{N}$. Let $\tau_{x}=\{\cos \alpha(s), \sin \alpha(s)\}$ be a tangent vector to $\Gamma$ in the point $x(s) \in \Gamma$, where $\cos \alpha(s)=x_{1}^{\prime}(s), \quad \sin \alpha(s)=x_{2}^{\prime}(s)$. Note that the parameter $s$ increases in the direction of the tangent vector $\tau_{x}$. Let $\mathbf{n}_{x}=\{\sin \alpha(s),-\cos \alpha(s)\}$ be the normal vector to $\Gamma$ at the point $x(s) \in \Gamma$. The normal vector $\mathbf{n}_{x}$ being rotated counterclockwise through an angle of $\pi / 2$ coincides with the tangent vector $\tau_{x}$. Let $\Gamma$ denote the set of segments $\bigcup_{n=1}^{N}\left[a_{n}, b_{n}\right]$ of the $0 s$ axis as well as the contour $\Gamma$.

Let the upper half-plane $R_{+}^{2}$ be slit along the contour $\Gamma$. Denote the side of the slits $\Gamma$, which remains on the left while the parameter $s$ increases, by $\Gamma^{+}$, and the opposite side by $\Gamma^{-}$. Let $X$ be the set of the ends of the contour $\Gamma: X=\bigcup_{n=1}^{N}\left(x\left(a_{n}\right) \cup x\left(b_{n}\right)\right)$.

We say that the function $p(x)$ belongs to the smoothness class $\mathcal{K}_{0}$ if the following conditions are satisfied:

(1) $p(x) \in C^{0}\left(\overline{R_{+}^{2} \backslash \Gamma} \backslash X\right) \cap C^{1}\left(R_{+}^{2} \backslash \Gamma\right)$, 
(2) for points $x \notin \Gamma$, lying in a small neighbourhood of any point $x(d) \in X$, the following inequality holds:

$$
|p(x)|<\mathcal{C}|x-x(d)|^{\varepsilon},
$$

where $\mathcal{C}>0$ and $\varepsilon>-1$ are some constants and $d=a_{n}$ or $d=b_{n}$ for $n=$ $1, \ldots, N$.

REmark. The class of functions continuous in $\overline{R_{+}^{2}} \backslash \Gamma$ and continuously extensible onto $\Gamma \backslash X$ from the left and from the right is denoted by $C^{0}\left(\overline{R_{+}^{2} \backslash \Gamma} \backslash X\right)$. The limiting values of these functions on $\Gamma \backslash X$ from the left and from the right can be different; i.e., these functions may have a jump if one passes across $\Gamma \backslash X$.

We say that the function $u(x)$ belongs to the smoothness class $\mathcal{K}$ if $u(x) \in C^{0}\left(\overline{R_{+}^{2}}\right) \cap$ $C^{2}\left(R_{+}^{2} \backslash \Gamma\right)$ and $\frac{\partial u(x)}{\partial x_{j}} \in \mathcal{K}_{0}$ for $j=1,2$.

Let us formulate the Dirichlet problem for the Stokes equation in the exterior of open arcs in the upper half-plane.

Problem $\mathcal{U}$. Find real functions $v_{1}(x), v_{2}(x) \in \mathcal{K}$, and $p(x) \in \mathcal{K}_{0}$, which obey the Stokes equations in $R_{+}^{2} \backslash \Gamma$,

$$
\begin{gathered}
k \Delta v_{j}-\frac{\partial p}{\partial x_{j}}=0, \quad j=1,2, \quad k=\text { const }>0, \quad \Delta=\left(\frac{\partial^{2}}{\partial x_{1}^{2}}+\frac{\partial^{2}}{\partial x_{2}^{2}}\right), \\
\frac{\partial v_{1}}{\partial x_{1}}+\frac{\partial v_{2}}{\partial x_{2}}=0
\end{gathered}
$$

satisfy the boundary conditions

$$
\begin{gathered}
\left.v_{1}(x)\right|_{x(s) \in \Gamma}=F_{1}(s),\left.\quad v_{2}(x)\right|_{x(s) \in \Gamma}=F_{2}(s), \\
\left.v_{1}(x)\right|_{x_{2}=0}=\left.v_{2}(x)\right|_{x_{2}=0}=0,
\end{gathered}
$$

and satisfy the following conditions if $x_{2}>0$ and $|x|=\sqrt{x_{1}^{2}+x_{2}^{2}} \rightarrow \infty$ :

$$
\left|v_{j}(x)\right|=o(1), \quad\left|\nabla v_{j}(x)\right|=o\left(|x|^{-1}\right), \quad j=1,2 ; \quad|p(x)|=o\left(|x|^{-1}\right) .
$$

All conditions of the problem must be satisfied in the classical sense. The edge condition (1) ensures the absence of point sources at the ends of $\Gamma$. The pressure in the fluid is denoted by $p(x)$, while components of the velocity vector are denoted by $v_{1}(x)$ and $v_{2}(x)$

We obtain problem $\mathcal{U}$ when studying the slow flow of viscous incompressible fluid filling an upper half-plane and bounded by a plane bottom $x_{2}=0$ over profiles $\Gamma$. It is assumed that the slow flow of viscous incompressible fluid obeys equations $(2 \mathrm{a}),(2 \mathrm{~b})$. Let the velocity vector be $\left(U_{0} x_{2}, 0\right)$ in the upstream flow as $x_{1} \rightarrow-\infty$, where $U_{0}$ is a positive constant; then the upstream flow satisfies both nonflow and nonslip conditions at the bottom, i.e. the upstream flow does not move near the bottom. The fluid in the upstream flow has to satisfy equations $(2 \mathrm{a}),(2 \mathrm{~b})$. Therefore, substituting the velocity vector $\left(U_{0} x_{2}, 0\right)$ into $(2 \mathrm{a}),(2 \mathrm{~b})$, we find that the pressure in the upstream flow equals an arbitrary constant $P_{0}$. Denote by $\left(U_{0} x_{2}+v_{1}(x), v_{2}(x)\right)$ the total velocity field in the perturbated flow over profiles $\Gamma$, where $\left(v_{1}(x), v_{2}(x)\right)$ is the perturbation of the velocity field. Denote by $P_{0}+p(x)$ the total pressure in the perturbated flow over $\Gamma$, where $p(x)$ 
is a perturbation of pressure. The total velocity field and the total pressure outside $\Gamma$ must satisfy equations $(2 \mathrm{a}),(2 \mathrm{~b})$. The velocity field $\left(U_{0} x_{2}, 0\right)$ and the pressure $P_{0}$ of nonperturbated flow obey equations $(2 \mathrm{a}),(2 \mathrm{~b})$. Therefore, these equations must be true for functions $v_{1}(x), v_{2}(x)$, and $p(x)$ as well. We assume that nonflow and nonslip conditions both hold on profiles $\Gamma$ and on the bottom $x_{2}=0$; i.e. $\left.\left(U_{0} x_{2}+v_{1}(x), v_{2}(x)\right)\right|_{\Gamma}=0$ and $\left.\left(U_{0} x_{2}+v_{1}(x), v_{2}(x)\right)\right|_{x_{2}=0}=0$. Then for the perturbation of the velocity field $\left(v_{1}(x), v_{2}(x)\right)$, we obtain boundary conditions $(2 \mathrm{~d}),(2 \mathrm{c})$, in which $F_{1}(s)=-U_{0} x_{2}(s)$ and $F_{2}(s)=0$, where $x=x(s) \in \Gamma$. From the physical standpoint, the perturbation of the velocity field and perturbation of pressure must vanish at infinity; this is assured by the conditions at infinity $(2 \mathrm{e})$. Thus, problem $\mathcal{U}$ describes the perturbation of the velocity field and perturbation of pressure when studying the creeping flow of viscous incompressible fluid over hard profiles $\Gamma$. It is known that for creeping flow (i.e. flow in the presence of the bottom), the Stokes paradox does not hold in a 2-D case; therefore, the problem being well-posed should have a solution, which will be constructed below. At first, we will study the uniqueness of the solution.

Below, by $\int_{\Gamma} \ldots d s$, we mean $\sum_{n=1}^{N} \int_{a_{n}}^{b_{n}} \ldots d s$. Using the method of integral equalities, one can prove

Theorem 1. If $\Gamma \in C^{2, \lambda}, \lambda \in(0,1]$, then problem $\mathcal{U}$ has no more than one solution.

Proof. Let $v_{1}^{0}(x), v_{2}^{0}(x), p^{0}(x)$ be an arbitrary solution of the homogeneous problem $\mathcal{U}$ (i.e. these functions belong to the required classes of smoothness and satisfy relations (2) with $\left.F_{1}(s) \equiv F_{2}(s) \equiv 0\right)$. Let us show that this solution is trivial. Consider a disc containing $\Gamma$ of sufficiently large radius $r$ and with the center in the origin. The part of the disc which is placed in the upper half-plane $x_{2}>0$, (i.e. upper half-disc) will be denoted by $C_{r}$. Denote by $\mathbf{n}_{x}$ the outward normal on $\partial C_{r}$. We envelope each slit $\Gamma_{n} \quad(n=1, \ldots, N)$ by the closed curve and write both the first Green's formula and the formula of integration by parts for $v_{j}^{0}(x) p_{x_{j}}^{0}(x) \quad(j=1,2)$ in the domain bounded by these curves and $\partial C_{r}$. Next we shrink the closed curves to the slits using smoothness of the solution of problem $\mathcal{U}$. Then these two formulae take the form

$$
\begin{gathered}
\int_{C_{r} \backslash \Gamma} v_{j}^{0} \Delta v_{j}^{0} d x=-\left\|\nabla v_{j}^{0}\right\|_{L_{2}\left(C_{r} \backslash \Gamma\right)}^{2}+\int_{\Gamma} v_{j}^{0}\left(\left(\frac{\partial v_{j}^{0}}{\partial \mathbf{n}_{x}}\right)^{+}-\left(\frac{\partial v_{j}^{0}}{\partial \mathbf{n}_{x}}\right)^{-}\right) d s \\
+\int_{\partial C_{r}} v_{j}^{0} \frac{\partial v_{j}^{0}}{\partial \mathbf{n}_{x}} d l, \\
\int_{C_{r} \backslash \Gamma} v_{j}^{0} \frac{\partial p^{0}}{\partial x_{j}} d x=\int_{\Gamma} v_{j}^{0}\left(\left(p^{0}\right)^{+}-\left(p^{0}\right)^{-}\right) \cos \left(\mathbf{n}_{x}, x_{j}\right) d s-\int_{C_{r} \backslash \Gamma} p^{0} \frac{\partial v_{j}^{0}}{\partial x_{j}} d x \\
+\int_{\partial C_{r}} v_{j}^{0} p^{0} \cos \left(\mathbf{n}_{x}, x_{j}\right) d l,
\end{gathered}
$$

where the condition (1) is used, $j=1,2$, and $\cos \left(\mathbf{n}_{x}, x_{j}\right)$ is the cosine of the angle between the normal vector $\mathbf{n}_{x}$ and the direction of the $O x_{j}$ axis. The curvilinear integral of the first kind is taken over $\partial C_{r}$. The superscripts + and - denote limiting values of functions on $\Gamma^{+}$and $\Gamma^{-}$, respectively. We multiply identity (3a) by $k$, subtract (3b), and take into 
account that functions $v_{j}^{0}(x)$ and $p^{0}(x)$ satisfy equations $(2 \mathrm{a})$ in $R_{+}^{2} \backslash \Gamma$; then we obtain the identity

$$
\begin{gathered}
\int_{C_{r} \backslash \Gamma} v_{j}^{0}\left(k \Delta v_{j}^{0} d x-\frac{\partial p^{0}}{\partial x_{j}}\right) d x \\
=-k\left\|\nabla v_{j}^{0}\right\|_{L_{2}\left(C_{r} \backslash \Gamma\right)}^{2}+\int_{C_{r} \backslash \Gamma} p^{0} \frac{\partial v_{j}^{0}}{\partial x_{j}} d x+k \int_{\Gamma} v_{j}^{0}\left(\left(\frac{\partial v_{j}^{0}}{\partial \mathbf{n}_{x}}\right)^{+}-\left(\frac{\partial v_{j}^{0}}{\partial \mathbf{n}_{x}}\right)^{-}\right) d s \\
-\int_{\Gamma} v_{j}^{0}\left(\left(p^{0}\right)^{+}-\left(p^{0}\right)^{-}\right) \cos \left(\mathbf{n}_{x}, x_{j}\right) d s \\
+k \int_{\partial C_{r}} v_{j}^{0} \frac{\partial v_{j}^{0}}{\partial \mathbf{n}_{x}} d l-\int_{\partial C_{r}} v_{j}^{0} p^{0} \cos \left(\mathbf{n}_{x}, x_{j}\right) d l=0, \quad j=1,2 .
\end{gathered}
$$

Adding these identities for $j=1$ and $j=2$ and using (2b), we obtain

$$
\begin{gathered}
-k\left(\left\|\nabla v_{1}^{0}\right\|_{L_{2}\left(C_{r} \backslash \Gamma\right)}^{2}+\left\|\nabla v_{2}^{0}\right\|_{L_{2}\left(C_{r} \backslash \Gamma\right)}^{2}\right) \\
+\sum_{j=1}^{2} \int_{\Gamma} v_{j}^{0}\left\{k\left(\left(\frac{\partial v_{j}^{0}}{\partial \mathbf{n}_{x}}\right)^{+}-\left(\frac{\partial v_{j}^{0}}{\partial \mathbf{n}_{x}}\right)^{-}\right)-\left(\left(p^{0}\right)^{+}-\left(p^{0}\right)^{-}\right) \cos \left(\mathbf{n}_{x}, x_{j}\right)\right\} d s \\
+\sum_{j=1}^{2} \int_{\partial C_{r}} v_{j}^{0}\left\{k \frac{\partial v_{j}^{0}}{\partial \mathbf{n}_{x}}-p^{0} \cos \left(\mathbf{n}_{x}, x_{j}\right)\right\} d l=0 .
\end{gathered}
$$

We set $r \rightarrow \infty$ in the latter identity and take into account (2e). Since the solution of the homogeneous problem satisfies zero boundary conditions (2c) and (2d), we obtain

$$
-k\left(\left\|\nabla v_{1}^{0}\right\|_{L_{2}\left(C_{r} \backslash \Gamma\right)}^{2}+\left\|\nabla v_{2}^{0}\right\|_{L_{2}\left(C_{r} \backslash \Gamma\right)}^{2}\right)=0 .
$$

Since $k>0$, we have

$$
\left\|\nabla v_{1}^{0}\right\|_{L_{2}\left(R_{+}^{2} \backslash \Gamma\right)}^{2}+\left\|\nabla v_{2}^{0}\right\|_{L_{2}\left(R_{+}^{2} \backslash \Gamma\right)}^{2}=0,
$$

whence $v_{1}^{0}(x) \equiv c_{1}$ and $v_{2}^{0}(x) \equiv c_{2}$ in $R_{+}^{2} \backslash \Gamma$, where $c_{1}$ and $c_{2}$ are some constants. These constants are equal to zero due to zero boundary conditions (2c) in the homogeneous problem $\mathcal{U}$; therefore, $v_{1}^{0}(x) \equiv v_{2}^{0}(x) \equiv 0$ in $R_{+}^{2}$. It follows from equations (2a) that $\nabla p^{0} \equiv 0$ in $R_{+}^{2} \backslash \Gamma$, whence $p^{0}(x) \equiv$ const in $R_{+}^{2} \backslash \Gamma$. Owing to conditions at infinity (2e): const $=0, p^{0}(x) \equiv 0$ in $R_{+}^{2} \backslash \Gamma$. Thus, the homogeneous problem $\mathcal{U}$ has only the trivial solution. Due to the linearity of problem $\mathcal{U}$, the inhomogeneous problem has at most one solution. The theorem is proved.

3. Elements of potential theory. We present some results of potential theory for Stokes equations in this section. These results will be used to solve problem $\mathcal{U}$.

Let $x=\left(x_{1}, x_{2}\right)$ be Cartesian coordinates in a plane $R^{2}$. Consider a simple smooth open arc $\gamma$ of class $C^{1, \lambda}, \quad \lambda \in(0,1]$, parametrized by the arc length $s: \gamma=\{x: x=$ $\left.x(s)=\left(x_{1}(s), x_{2}(s)\right), s \in[a, b]\right\}$. The tangent vector to $\gamma$ in the point $x(s)$ directed to the increment of parameter $s$ will be denoted by $\tau_{s}=(\cos \alpha(s), \sin \alpha(s))$, while the normal vector coinciding with $\tau_{s}$ after counterclockwise rotation through an angle of $\pi / 2$ will be denoted by $\mathbf{n}_{s}=(\sin \alpha(s),-\cos \alpha(s))$. The chosen parametrization implies that 
$\cos \alpha(s)=x_{1}^{\prime}(s), \sin \alpha(s)=x_{2}^{\prime}(s)$. The interval $[a, b]$ on the $O s$ axis will be denoted by $\gamma$ as well.

Let $\mathcal{F}(s)$ be a function specified on $[a, b]$. We say that $\mathcal{F}(s) \in C_{q}^{\omega}[a, b]$ if $\mathcal{F}_{0}(s) \in$ $C^{0, \omega}[a, b]$, where $\mathcal{F}_{0}(s)=\mathcal{F}(s)|s-a|^{q}|s-b|^{q}$, and $C^{0, \omega}[a, b]$ is a class of Hölder continuous functions with the exponent $\omega$.

Consider $\gamma$ as a slit in a plane. The side of the slit $\gamma$ which is positioned on the left as parameter $s$ increases will be denoted by $\gamma^{+}$, while the opposite side will be denoted by $\gamma^{-}$. Let $\mu_{1}(s), \mu_{2}(s)$ be integrable functions specified on $[a, b]$. We introduce the column $\mu(s)=\left(\mu_{1}(s), \mu_{2}(s)\right)^{T}$. Let us study velocity potentials [1, 3, 8] for Stokes equations (2a), $(2 \mathrm{~b})$. These potentials can be written in the form:

$$
\begin{gathered}
V_{1}[\mu](x)=\frac{1}{4 \pi k}\left\{-\int_{\gamma} \mu_{1}(\sigma) \ln |x-y(\sigma)| d \sigma+\int_{\gamma} \mu_{1}(\sigma) \frac{1+\cos (2 \psi(x, y(\sigma)))}{2} d \sigma\right. \\
\left.+\int_{\gamma} \mu_{2}(\sigma) \frac{\sin (2 \psi(x, y(\sigma)))}{2} d \sigma\right\}, \quad V_{2}[\mu](x)=\frac{1}{4 \pi k}\left\{\int_{\gamma} \mu_{1}(\sigma) \frac{\sin (2 \psi(x, y(\sigma)))}{2} d \sigma\right. \\
\left.-\int_{\gamma} \mu_{2}(\sigma) \ln |x-y(\sigma)| d \sigma+\int_{\gamma} \mu_{2}(\sigma) \frac{1-\cos (2 \psi(x, y(\sigma)))}{2} d \sigma\right\}
\end{gathered}
$$

where $k=$ const, $\quad \cos \psi(x, y(\sigma))=\frac{x_{1}-y_{1}(\sigma)}{|x-y(\sigma)|}, \quad \sin \psi(x, y(\sigma))=\frac{x_{2}-y_{2}(\sigma)}{|x-y(\sigma)|}$,

$$
y(\sigma)=\left(y_{1}(\sigma), y_{2}(\sigma)\right) \in \gamma, \quad|x-y(\sigma)|=\sqrt{\left(x_{1}-y_{1}(\sigma)\right)^{2}+\left(x_{2}-y_{2}(\sigma)\right)^{2}},
$$

and the formulae used are $\cos (2 \psi)=2 \cos ^{2}(\psi)-1=1-2 \sin ^{2}(\psi), \sin (2 \psi)=2 \sin \psi \cos \psi$. The pressure potential related to the above velocity potentials takes the form

$$
P[\mu](x)=\frac{1}{2 \pi} \int_{\Gamma} \mu_{1}(\sigma) \frac{\cos (\psi(x, y(\sigma)))}{|x-y(\sigma)|} d \sigma+\frac{1}{2 \pi} \int_{\Gamma} \mu_{2}(\sigma) \frac{\sin (\psi(x, y(\sigma)))}{|x-y(\sigma)|} d \sigma .
$$

The following theorem holds $[\underline{8}$.

Theorem 2. Let $\gamma$ be a simple open arc of class $C^{1, \lambda}, \lambda \in(0,1]$. Let $\mu_{1}(\sigma), \mu_{2}(\sigma) \in$ $C_{q}^{\omega}[a, b], \omega \in(0,1], q \in[0,1)$. Then

1) the functions $V_{1}[\mu](x), V_{2}[\mu](x)$ belong to $C^{0}\left(R^{2}\right) \cap C^{2}\left(R^{2} \backslash \gamma\right)$, while $\nabla V_{1}[\mu](x)$ and $\nabla V_{2}[\mu](x)$ belong to $C^{0}\left(\overline{R^{2} \backslash \gamma} \backslash X_{\gamma}\right)$, where $X_{\gamma}=x(a) \cup x(b)$ is a set of endpoints of $\gamma$; 
2) the limiting values of normal and tangent derivatives of potentials $V_{1}[\mu](x)$ and $V_{2}[\mu](x)$ on $\gamma^{ \pm} \backslash X_{\gamma}$ are given by formulae

$$
\begin{aligned}
& \left.\frac{\partial V_{1}[\mu](x)}{\partial \tau_{x}}\right|_{x=x(s) \in \gamma^{ \pm}}=\frac{\partial V_{1}[\mu](x(s))}{\partial s}=\frac{1}{4 \pi}\left\{-\int_{\gamma} \mu_{1}(\sigma) \frac{\cos (\psi(x, y(\sigma))-\alpha(s))}{|x-y(\sigma)|} d \sigma\right. \\
& +\int_{\gamma} \mu_{1}(\sigma) \sin (2 \psi(x, y(\sigma))) \frac{\sin (\psi(x, y(\sigma))-\alpha(s))}{|x-y(\sigma)|} d \sigma \\
& \left.-\int_{\gamma} \mu_{2}(\sigma) \cos (2 \psi(x, y(\sigma))) \frac{\sin (\psi(x, y(\sigma))-\alpha(s))}{|x-y(\sigma)|} d \sigma\right\}, \\
& \left.\frac{\partial V_{2}[\mu](x)}{\partial \tau_{x}}\right|_{x=x(s) \in \gamma^{ \pm}}=\frac{\partial V_{2}[\mu](x(s))}{\partial s} \\
& =\frac{1}{4 \pi k}\left\{-\int_{\gamma} \mu_{1}(\sigma) \cos (2 \psi(x, y(\sigma))) \frac{\sin (\psi(x, y(\sigma))-\alpha(s))}{|x-y(\sigma)|} d \sigma\right. \\
& -\int_{\gamma} \mu_{2}(\sigma) \frac{\cos (\psi(x, y(\sigma))-\alpha(s))}{|x-y(\sigma)|} d \sigma \\
& \left.-\int_{\gamma} \mu_{2}(\sigma) \sin (2 \psi(x, y(\sigma))) \frac{\sin (\psi(x, y(\sigma))-\alpha(s))}{|x-y(\sigma)|} d \sigma\right\} \\
& \left.\frac{\partial V_{1}[\mu](x)}{\partial \mathbf{n}_{x}}\right|_{x=x(s) \in \gamma^{ \pm}}=\frac{1}{4 \pi k}\left\{ \pm \pi \mu_{1}(s)(1+\cos (2 \alpha(s)))\right. \\
& +\int_{\gamma} \mu_{1}(\sigma) \frac{\sin (\psi(x, y(\sigma))-\alpha(s))}{|x-y(\sigma)|} d \sigma \\
& +\int_{\gamma} \mu_{1}(\sigma) \sin (2 \psi(x, y(\sigma))) \frac{\cos (\psi(x, y(\sigma))-\alpha(s))}{|x-y(\sigma)|} d \sigma \pm \pi \mu_{2}(s) \sin (2 \alpha(s)) \\
& \left.-\int_{\gamma} \mu_{2}(\sigma) \cos (2 \psi(x, y(\sigma))) \frac{\cos (\psi(x, y(\sigma))-\alpha(s))}{|x-y(\sigma)|} d \sigma\right\}, \\
& \left.\frac{\partial V_{2}[\mu](x)}{\partial \mathbf{n}_{x}}\right|_{x=x(s) \in \gamma^{ \pm}}=\frac{1}{4 \pi k}\left\{ \pm \pi \mu_{1}(s) \sin (2 \alpha(s))\right. \\
& -\int_{\gamma} \mu_{1}(\sigma) \cos (2 \psi(x, y(\sigma))) \frac{\cos (\psi(x, y(\sigma))-\alpha(s))}{|x-y(\sigma)|} d \sigma \pm \pi \mu_{2}(s)(1-\cos (2 \alpha(s))) \\
& +\int_{\gamma} \mu_{2}(\sigma) \frac{\sin (\psi(x, y(\sigma))-\alpha(s))}{|x-y(\sigma)|} d \sigma \\
& \left.-\int_{\gamma} \mu_{2}(\sigma) \sin (2 \psi(x, y(\sigma))) \frac{\cos (\psi(x, y(\sigma))-\alpha(s))}{|x-y(\sigma)|} d \sigma\right\},
\end{aligned}
$$

where the integrals containing $\cos (\psi(x, y(\sigma))-\alpha(s))$ are understood in the sense of principal value;

3) for any point $x \notin \gamma$ placed in the small neighbourhood of the end $x(d)$ ( $d=a$ or $d=b)$, the inequalities hold:

$$
\left|\frac{\partial V_{1}[\mu](x)}{\partial x_{j}}\right| \leq \frac{\text { const }}{|x-x(d)|^{\delta}}, \quad\left|\frac{\partial V_{2}[\mu](x)}{\partial x_{j}}\right| \leq \frac{\text { const }}{|x-x(d)|^{\delta}}, \quad j=1,2,
$$

where $\delta=q$ if $q \in(0,1)$, and $\delta$ is an arbitrary number from the interval $(0,1)$ if $q=0$. 
REMARK 1. The class of functions, which are continuous outside $\gamma$ and are continuously extensible on the sides of the slit $\gamma \backslash X_{\gamma}$ from the left and the right, is denoted by $C^{0}\left(\overline{R^{2} \backslash \gamma} \backslash X_{\gamma}\right)$. Note that the limiting values of such a function on $\gamma$ from the left and the right can be different; i.e. such a function may have a jump when one passes across $\gamma \backslash X_{\gamma}$.

REmark 2. The potentials $V_{1}[\mu](x), V_{2}[\mu](x)$ were studied in [1, 3] with the assumption that the densities $\mu_{1}(\sigma), \mu_{2}(\sigma)$ are continuous on the curve on integration. The formulae from point 2 of Theorem 2 does not hold under this assumption in general, since the singular integrals in the formulae may not exist, so the formulae were not derived in [1, 3].

Theorem 2 straightforwardly follows from results presented in [6, 9. Properties of the pressure potential $P[\mu](x)$ are presented in [7] and [9, Section 8].

4. Integral equations at the boundary. To construct the solution of problem $\mathcal{U}$, we assume that the functions $F_{1}(s), F_{2}(s)$ in the boundary conditions $(2 \mathrm{c})$ possess the following smoothness:

$$
F_{1}(s), F_{2}(s) \in C^{1, \lambda}(\Gamma), \quad \lambda \in(0,1] .
$$

Note that here, the Hölder exponent $\lambda$ is assumed to be the same as in the definition of the smoothness of the arcs $\Gamma$. If in (4) and in the definition of the smoothness of the arcs $\Gamma$ these exponents are different, then as $\lambda$ we may take the least.

We will construct the solution of problem $\mathcal{U}$ by the method of potentials. Set $r\left(x, y_{1}, y_{2}\right)=\sqrt{\left(x_{1}-y_{1}\right)^{2}+\left(x_{2}-y_{2}\right)^{2}}=|x-y|$,

$$
\begin{gathered}
\cos \left(\psi\left(x, y_{1}, y_{2}\right)\right)=\frac{x_{1}-y_{1}}{r\left(x, y_{1}, y_{2}\right)}=\cos (\psi(x, y)), \\
\sin \left(\psi\left(x, y_{1}, y_{2}\right)\right)=\frac{x_{2}-y_{2}}{r\left(x, y_{1}, y_{2}\right)}=\sin (\psi(x, y)), \\
L_{l l}\left(x, y_{1}, y_{2}\right)=-\ln r\left(x, y_{1}, y_{2}\right)+\frac{1-(-1)^{l} \cos \left(2 \psi\left(x, y_{1}, y_{2}\right)\right)}{2}, \quad l=1,2, \\
L_{12}\left(x, y_{1}, y_{2}\right)=L_{21}\left(x, y_{1}, y_{2}\right)=\frac{\sin \left(2 \psi\left(x, y_{1}, y_{2}\right)\right)}{2}, \\
H_{1}(x, y)=2 x_{2} y_{2} \frac{\cos \left(2 \psi\left(x, y_{1},-y_{2}\right)\right)}{\left(r\left(x, y_{1},-y_{2}\right)\right)^{2}}, \quad H_{2}(x, y)=2 x_{2} y_{2} \frac{\sin \left(2 \psi\left(x, y_{1},-y_{2}\right)\right)}{\left(r\left(x, y_{1},-y_{2}\right)\right)^{2}}, \\
H_{3}(x, y)=2 y_{2} \frac{\cos \left(\psi\left(x, y_{1},-y_{2}\right)\right)}{r\left(x, y_{1},-y_{2}\right)} .
\end{gathered}
$$

To find $\cos (2 \psi), \sin (2 \psi)$, we use formulae

$$
\sin (2 \psi)=2 \sin \psi \cos \psi, \cos (2 \psi)=2 \cos ^{2}(\psi)-1=1-2 \sin ^{2}(\psi) .
$$

Let $g_{1}(s), g_{2}(s)$ be real integrable functions defined on $\Gamma$. Potentials of velocities and potential of pressure for equations $(2 \mathrm{a}),(2 \mathrm{~b})$ are presented in 1, 3 (see also Section 3 and [7]). Potentials of velocities satisfying condition (2d) can be constructed by the 
method of reflection and are given by formulae:

$$
\begin{gathered}
v_{1}\left[g_{1}, g_{2}\right](x) \\
=\frac{1}{4 \pi k} \int_{\Gamma}\left\{g_{1}(\sigma)\left[L_{11}\left(x, y_{1}(\sigma), y_{2}(\sigma)\right)-L_{11}\left(x, y_{1}(\sigma),-y_{2}(\sigma)\right)+H_{1}(x, y(\sigma))\right]\right. \\
\left.+g_{2}(\sigma)\left[L_{12}\left(x, y_{1}(\sigma), y_{2}(\sigma)\right)-L_{12}\left(x, y_{1}(\sigma),-y_{2}(\sigma)\right)-H_{2}(x, y(\sigma))+H_{3}(x, y(\sigma))\right]\right\} d \sigma, \\
v_{2}\left[g_{1}, g_{2}\right](x) \\
=\frac{1}{4 \pi k} \int_{\Gamma}\left\{g_{1}(\sigma)\left[L_{21}\left(x, y_{1}(\sigma), y_{2}(\sigma)\right)-L_{21}\left(x, y_{1}(\sigma),-y_{2}(\sigma)\right)+H_{2}(x, y(\sigma))+H_{3}(x, y(\sigma))\right]\right. \\
\left.\quad+g_{2}(\sigma)\left[L_{22}\left(x, y_{1}(\sigma), y_{2}(\sigma)\right)-L_{22}\left(x, y_{1}(\sigma),-y_{2}(\sigma)\right)+H_{1}(x, y(\sigma))\right]\right\} d \sigma,
\end{gathered}
$$

where $k$ is a constant from (2a). The corresponding pressure potential is

$$
\begin{aligned}
& p\left[g_{1}, g_{2}\right](x) \\
= & \frac{1}{2 \pi} \int_{\Gamma}\left\{g_{1}(\sigma)\left(\frac{\cos (\psi(x, y(\sigma))}{|x-y(\sigma)|}-\frac{\cos \left(\psi\left(x, y_{1}(\sigma),-y_{2}(\sigma)\right)\right)}{r\left(x, y_{1}(\sigma),-y_{2}(\sigma)\right)}+\frac{1}{x_{2}} H_{2}(x, y(\sigma))\right)\right. \\
& \left.+g_{2}(\sigma)\left(\frac{\sin (\psi(x, y(\sigma)))}{|x-y(\sigma)|}-\frac{\sin \left(\psi\left(x, y_{1}(\sigma),-y_{2}(\sigma)\right)\right)}{r\left(x, y_{1}(\sigma),-y_{2}(\sigma)\right)}+\frac{1}{x_{2}} H_{1}(x, y(\sigma))\right)\right\} d \sigma .
\end{aligned}
$$

Properties of potentials for the half-plane follow from properties of potentials for the whole plane (see [1, 3, 7, and Section 3). It follows from [1, 3, that for any integrable functions $g_{1}(s), g_{2}(s)$, the potentials $v_{1}\left[g_{1}, g_{2}\right](x), v_{2}\left[g_{1}, g_{2}\right](x), p\left[g_{1}, g_{2}\right](x)$ satisfy equations (2a), (2b) in $R_{+}^{2} \backslash \Gamma$. It can be verified directly that for any integrable functions $g_{1}(s)$, $g_{2}(s)$, these potentials satisfy conditions at infinity (2e) and condition (2d) as well. In particular, the potentials demonstrate the following behaviour as $|x|=\sqrt{x_{1}^{2}+x_{2}^{2}} \rightarrow \infty$ :

$$
\begin{gathered}
p\left[g_{1}, g_{2}\right](x)=O\left(|x|^{-2}\right) ; \\
v_{j}\left[g_{1}, g_{2}\right](x)=O\left(|x|^{-1}\right), \quad \frac{\partial v_{j}\left[g_{1}, g_{2}\right](x)}{\partial x_{l}}=O\left(|x|^{-2}\right), \quad j, l=1,2 .
\end{gathered}
$$

We say that the function $\Phi(s)$ belongs to the space $C_{q}^{\omega}(\Gamma)$ with $\omega \in(0,1], q \in[0,1)$ if $\Phi(s) \prod_{n=1}^{N}\left|s-a_{n}\right|^{q}\left|s-b_{n}\right|^{q} \in C^{0, \omega}(\Gamma)$, where $C^{0, \omega}(\Gamma)$ is a space of Hölder continuous functions with the exponent $\omega$. The norm in the Banach space $C_{q}^{\omega}(\Gamma)$ is defined by the formula $\|\Phi(s)\|_{C_{q}^{\omega}(\Gamma)}=\left\|\Phi(s) \prod_{n=1}^{N}\left|s-a_{n}\right|^{q}\left|s-b_{n}\right|^{q}\right\|_{C^{0, \omega}(\Gamma)}$.

Further on, we assume that densities $g_{1}(\sigma), g_{2}(\sigma)$ belong to the Banach space $C_{q}^{\omega}(\Gamma)$ for some $\omega, q$, so that $\omega \in(0,1], q \in[0,1)$.

It follows from Theorem 2 and from [7] that for such densities $g_{1}(\sigma), g_{2}(\sigma)$, the potentials of velocities $v_{1}\left[g_{1}, g_{2}\right](x), v_{2}\left[g_{1}, g_{2}\right](x)$ belong to the class $\mathcal{K}$, while the potential of pressure $p\left[g_{1}, g_{2}\right](x)$ belongs to the class $\mathcal{K}_{0}$.

We will look for the solution of problem $\mathcal{U}$ in the form

$$
\left\{v_{1}\left[g_{1}, g_{2}\right](x), v_{2}\left[g_{1}, g_{2},\right](x), p\left[g_{1}, g_{2}\right](x)\right\} ;
$$

i.e. we look for velocities and for pressure in the form of corresponding potentials for velocities and for pressure from (6), (7). 
It was mentioned above that the densities $g_{1}(s), g_{2}(s)$ will be sought in the space $C_{q}^{\omega}(\Gamma)$ with $\omega \in(0,1], q \in[0,1)$. It follows from the above arguments that for such densities $g_{1}(s), g_{2}(s)$, the functions $v_{1}\left[g_{1}, g_{2}\right](x), v_{2}\left[g_{1}, g_{2}\right](x)$ and $p\left[g_{1}, g_{2}\right](x)$ satisfy all conditions of problem $\mathcal{U}$ except for boundary conditions $(2 \mathrm{c})$.

To satisfy boundary conditions (2c), we substitute (6) into (2c) and obtain two integral equations for densities $g_{1}(s), g_{2}(s)$ :

$$
v_{1}\left[g_{1}, g_{2}\right](x(s))=F_{1}(s), \quad v_{2}\left[g_{1}, g_{2}\right](x(s))=F_{2}(s), \quad s \in \Gamma,
$$

where $v_{1}\left[g_{1}, g_{2}\right](x), v_{2}\left[g_{1}, g_{2}\right](x)$ are potentials of velocities from (6), in which we have to set $x=x(s)$. Differentiating each equation (8) in $s$ (see Theorem 2), we obtain two singular integral equations with respect to functions $g_{1}(s), g_{2}(s)$ :

$$
\begin{gathered}
\frac{\partial v_{1}\left[g_{1}, g_{2}\right](x(s))}{\partial s}=\frac{1}{4 \pi k} \int_{\Gamma}\left\{g _ { 1 } ( \sigma ) \left(-\frac{\cos (\psi(x(s), y(\sigma))-\alpha(s))}{|x(s)-y(\sigma)|}\right.\right. \\
+\sin (2 \psi(x(s), y(\sigma))) \frac{\sin (\psi(x(s), y(\sigma))-\alpha(s))}{|x(s)-y(\sigma)|}-\frac{\partial L_{11}\left(x(s), y_{1}(\sigma),-y_{2}(\sigma)\right)}{\partial s} \\
\left.+\frac{\partial H_{1}(x(s), y(\sigma))}{\partial s}\right)+g_{2}(\sigma)\left(-\cos (2 \psi(x(s), y(\sigma))) \frac{\sin (\psi(x(s), y(\sigma))-\alpha(s))}{|x(s)-y(\sigma)|}\right. \\
\left.\left.-\frac{\partial L_{12}\left(x(s), y_{1}(\sigma),-y_{2}(\sigma)\right)}{\partial s}-\frac{\partial H_{2}(x(s), y(\sigma))}{\partial s}+\frac{\partial H_{3}(x(s), y(\sigma))}{\partial s}\right)\right\} d \sigma \\
=\frac{d F_{1}(s)}{d s}=F_{1}^{\prime}(s), \quad s \in \Gamma, \\
-\sin (2 \psi(x(s), y(\sigma))) \frac{\sin (\psi(x(s), y(\sigma))-\alpha(s))}{|x(s)-y(\sigma)|}-\frac{\partial L_{22}\left(x(s), y_{1}(\sigma),-y_{2}(\sigma)\right)}{\partial s} \\
\left.+\frac{\partial H_{1}(x(s), y(\sigma))}{\partial s}\right)+g_{1}(\sigma)\left(-\cos (2 \psi(x(s), y(\sigma))) \frac{\sin (\psi(x(s), y(\sigma))-\alpha(s))}{|x(s)-y(\sigma)|}\right. \\
\left.\left.-\frac{\partial L_{21}\left(x(s), y_{1}(\sigma),-y_{2}(\sigma)\right)}{\partial s}+\frac{\partial H_{2}(x(s), y(\sigma))}{\partial s}+\frac{\partial H_{3}(x(s), y(\sigma))}{\partial s}\right)\right\} d \sigma \\
=\frac{d F_{2}(s)}{d s}=F_{2}^{\prime}(s), \quad s \in \Gamma .
\end{gathered}
$$

The first terms in equations (9a) and (9b) are Cauchy singular integrals. Functions $L_{j l}\left(x, y_{1},-y_{2}\right)$ with $j, l=1,2$ and $H_{m}(x, y)$ with $m=1,2,3$ are defined by formulae (5), while $\alpha(s)$ is an angle of inclination of the tangent vector $\tau_{x}$ to the $0 x_{1}$ axis. In order that equations (9) be equivalent to equations (8), it is necessary to supply equations (9) with the following additional conditions:

$$
v_{1}\left[g_{1}, g_{2}\right]\left(x\left(a_{n}\right)\right)=F_{1}\left(a_{n}\right), \quad v_{2}\left[g_{1}, g_{2}\right]\left(x\left(a_{n}\right)\right)=F_{2}\left(a_{n}\right), \quad n=1, \ldots, N .
$$

From the above arguments follows

Theorem 3. Let $\Gamma \in C^{2, \lambda}, F_{1}(s), F_{2}(s) \in C^{1, \lambda}(\Gamma), \lambda \in(0,1]$. If the system of equations (9), (10) has a solution $\left\{g_{1}(s), g_{2}(s)\right\}$ such that $g_{1}(s), g_{2}(s) \in C_{q}^{\omega}(\Gamma)$ with $\omega \in(0,1]$, 
$q \in[0,1)$, then the solution $\left\{v_{1}\left[g_{1}, g_{2}\right](x), v_{2}\left[g_{1}, g_{2}\right](x), p\left[g_{1}, g_{2}\right](x)\right\}$ of problem $\mathcal{U}$ exists and is given by the formulae (6), (7).

Below we prove that the system (9), (10) has a solution $\left\{g_{1}(s), g_{2}(s)\right\}$ such that $g_{1}(s), g_{2}(s) \in C_{q}^{\omega}(\Gamma)$ with $\omega \in(0,1], q \in[0,1)$. Moreover, we reduce the system (9), (10) to the uniquely solvable Fredholm integral equation of the second kind and with index zero. This Fredholm integral equation can be solved numerically by standard codes.

It is shown in [6, lemmas 2, 3] that

$$
\begin{gathered}
\left(\frac{-\cos [\psi(x(s), y(\sigma))-\alpha(s)]}{|x(s)-y(\sigma)|}-\frac{1}{\sigma-s}\right) \in C^{0, \lambda}(\Gamma \times \Gamma), \\
\frac{\sin (\psi(x(s), y(\sigma))-\alpha(s))}{|x(s)-y(\sigma)|} \in C^{0, \lambda}(\Gamma \times \Gamma),
\end{gathered}
$$

since $\Gamma \in C^{2, \lambda}$. Besides, according to 9 ,

$$
\cos (2 \psi(x(s), y(\sigma))), \sin (2 \psi(x(s), y(\sigma))) \in C^{0,1}(\Gamma \times \Gamma) .
$$

It can be shown that $\frac{\partial L_{j l}\left(x(s), y_{1}(\sigma),-y_{2}(\sigma)\right)}{\partial s}, \frac{\partial H_{m}(x(s), y(\sigma))}{\partial s} \in C^{1}(\Gamma \times \Gamma)$ for $j, l=$ 1,2 and $m=1,2,3$, since the functions $L_{j l}\left(x, y_{1},-y_{2}\right)$ with $j, l=1,2$ and functions $H_{m}(x, y)$ with $m=1,2,3$ do not have singularity as $x \rightarrow y \in \Gamma$. Consequently, we may rewrite equations (9) in the form

$$
\begin{aligned}
& \frac{1}{\pi} \int_{\Gamma} g_{1}(\sigma) \frac{d \sigma}{\sigma-s}+\sum_{l=1}^{2} \int_{\Gamma} Y_{1 l}(s, \sigma) g_{l}(\sigma) d \sigma=4 k F_{1}^{\prime}(s), \quad s \in \Gamma, \\
& \frac{1}{\pi} \int_{\Gamma} g_{2}(\sigma) \frac{d \sigma}{\sigma-s}+\sum_{l=1}^{2} \int_{\Gamma} Y_{2 l}(s, \sigma) g_{l}(\sigma) d \sigma=4 k F_{2}^{\prime}(s), \quad s \in \Gamma,
\end{aligned}
$$

where

$$
\begin{gathered}
Y_{l l}(s, \sigma)=\frac{1}{\pi}\left\{\left(\frac{-\cos [\psi(x(s), y(\sigma))-\alpha(s)]}{|x(s)-y(\sigma)|}-\frac{1}{\sigma-s}\right)\right. \\
-(-1)^{l} \sin \left(2 \psi(x(s), y(\sigma)) \frac{\sin (\psi(x(s), y(\sigma))-\alpha(s))}{|x(s)-y(\sigma)|}\right. \\
\left.-\frac{\partial L_{l l}\left(x(s), y_{1}(\sigma),-y_{2}(\sigma)\right)}{\partial s}+\frac{\partial H_{1}(x(s), y(\sigma))}{\partial s}\right\}, \quad l=1,2, \\
Y_{12}(s, \sigma)=\frac{1}{\pi}\left\{-\cos (2 \psi(x(s), y(\sigma))) \frac{\sin (\psi(x(s), y(\sigma))-\alpha(s))}{|x(s)-y(\sigma)|}\right. \\
\left.-\frac{\partial L_{12}\left(x(s), y_{1}(\sigma),-y_{2}(\sigma)\right)}{\partial s}-\frac{\partial H_{2}(x(s), y(\sigma))}{\partial s}+\frac{\partial H_{3}(x(s), y(\sigma))}{\partial s}\right\}, \\
Y_{21}(s, \sigma)=Y_{12}(s, \sigma)+\frac{2}{\pi} \frac{\partial H_{2}(x(s), y(\sigma))}{\partial s},
\end{gathered}
$$

and $Y_{j l}(s, \sigma) \in C^{0, \lambda}(\Gamma \times \Gamma), \lambda \in(0,1]$, for $j, l=1,2$. Here we took into account that $L_{12}\left(x(s), y_{1}(\sigma),-y_{2}(\sigma)\right)=L_{21}\left(x(s), y_{1}(\sigma),-y_{2}(\sigma)\right)$, owing to $(5)$. 
5. Fredholm integral equation and the solution of the problem. Inverting a singular integral operator in the equations (11), we obtain two integral equations of the second kind [10:

$$
\begin{gathered}
g_{1}(s)-\frac{1}{Q(s)} \sum_{l=1}^{2} \int_{\Gamma} g_{l}(\sigma) A_{1 l}(s, \sigma) d \sigma+\frac{1}{Q(s)} \sum_{n=0}^{N-1} G_{n} s^{n}=\frac{f_{1}(s)}{Q(s)}, \quad s \in \Gamma, \\
g_{2}(s)-\frac{1}{Q(s)} \sum_{l=1}^{2} \int_{\Gamma} g_{l}(\sigma) A_{2 l}(s, \sigma) d \sigma+\frac{1}{Q(s)} \sum_{n=0}^{N-1} G_{n+N} s^{n}=\frac{f_{2}(s)}{Q(s)}, \quad s \in \Gamma,
\end{gathered}
$$

where

$$
\begin{gathered}
A_{j l}(s, \sigma)=\frac{1}{\pi} \int_{\Gamma} \frac{Y_{j l}(\xi, \sigma)}{\xi-s} Q(\xi) d \xi, \quad j=1,2, \quad l=1,2, \\
Q(s)=\prod_{n=1}^{N}\left|\sqrt{s-a_{n}} \sqrt{b_{n}-s}\right| \operatorname{sign}\left(s-a_{n}\right), \\
f_{j}(s)=-\frac{4 k}{\pi} \int_{\Gamma} \frac{Q(\xi) F_{j}^{\prime}(\xi)}{\xi-s} d \xi, \quad j=1,2,
\end{gathered}
$$

and $G_{0}, \ldots, G_{2 N-1}$ are arbitrary constants, which must be defined when solving the problem. It is assumed that $\operatorname{sign}\left(s-a_{n}\right)=1$ if $s=a_{n}$; then $\operatorname{sign}\left(s-a_{n}\right)$ belongs to $C^{\infty}(\Gamma)$ in variable $s$ for $n=1, \ldots, N$.

Note that if functions $g_{1}(s), g_{2}(s) \in C_{q}^{\omega}(\Gamma)$ satisfy equations (12) for certain constants $G_{0}, \ldots, G_{2 N-1}$, then they also satisfy equations (11). (This can be easily shown if we apply the singular integral operator with the kernel $(\sigma-s)^{-1}$ to equations (12); then we obtain equations (11).)

Let us study properties of functions in (12). The densities of singular integrals in expressions for functions $A_{j l}(s, \sigma), f_{j}(s) \quad(j, l=1,2)$ are Hölder continuous on $\Gamma$, and densities in $A_{j l}(s, \sigma)$ are Hölder continuous in both variables. In particular, these densities are Hölder continuous on $\Gamma$ in variable $\xi$ with the exponent $\beta=\min \{\lambda, 1 / 2\}$ (uniformly with respect to $\sigma$ in the case of $A_{j l}(s, \sigma)$ ) and are equal to zero if $\xi$ is an end of $\Gamma$ (since the function $Q(\xi)$ belongs to the class $C^{0,1 / 2}(\Gamma)$ and equals zero at the ends of $\Gamma$ ). Using properties of singular integrals [10, §18], we arrive at

Lemma 1. Let $\Gamma \in C^{2, \lambda}(\Gamma), \lambda \in(0,1]$. Then functions $A_{j l}(s, \sigma)$ with $j=1,2, l=1,2$ are Hölder continuous on $\Gamma$ in both variables. In particular, these functions are Hölder continuous on $\Gamma$ in variable $s$ with the exponent $\beta=\min \{\lambda, 1 / 2\}$ uniformly with respect to $\sigma \in \Gamma$. If, in addition, conditions (4) hold, then $f_{1}(s), f_{2}(s) \in C^{0, \beta}(\Gamma)$.

It follows from Lemma 1 that integrals in (12) are Hölder continuous in $s$ on $\Gamma$ with the exponent $\beta=\min \{\lambda, 1 / 2\}$ for any functions $g_{1}(s), g_{2}(s)$ from the space $C_{q}^{\omega}(\Gamma)$ with $\omega \in(0,1]$ and $q \in[0,1)$. It can be verified directly using Lemma 1 that if functions $g_{1}(s), g_{2}(s)$ of the space $C_{q}^{\omega}(\Gamma)$ with $\omega \in(0,1]$ and $q \in[0,1)$ satisfy equations (12), then $g_{1}(s), g_{2}(s) \in C_{1 / 2}^{\beta}(\Gamma)$ with $\beta=\min \{\lambda, 1 / 2\}$. Therefore, we will look for functions $g_{1}(s)$, $g_{2}(s)$ in the space $C_{1 / 2}^{\beta}(\Gamma)$ with $\beta=\min \{\lambda, 1 / 2\}$ below. 
Instead of functions $g_{1}(s), g_{2}(s) \in C_{1 / 2}^{\beta}(\Gamma)$, we introduce the new unknown functions $g_{1 *}(s), g_{2 *}(s) \in C^{0, \beta}(\Gamma)$ by the formula $g_{j *}(s)=g_{j}(s) Q(s), j=1,2$ and rewrite (12) in the form

$$
\begin{gathered}
g_{1 *}(s)-\sum_{l=1}^{2} \int_{\Gamma} A_{1 l}(s, \sigma) g_{l *}(\sigma) Q^{-1}(\sigma) d \sigma+\sum_{n=0}^{N-1} G_{n} s^{n}=f_{1}(s), \quad s \in \Gamma, \\
g_{2 *}(s)-\sum_{l=1}^{2} \int_{\Gamma} A_{2 l}(s, \sigma) g_{l *}(\sigma) Q^{-1}(\sigma) d \sigma+\sum_{n=0}^{N-1} G_{n+N} s^{n}=f_{2}(s), \quad s \in \Gamma .
\end{gathered}
$$

We rewrite conditions (10) in terms of functions $g_{1 *}(s), g_{2 *}(s)$ :

$$
\begin{gathered}
\mathcal{L}_{n, 1} g_{1 *}+\mathcal{L}_{n, 2} g_{2 *}=F_{1}\left(a_{n}\right), \quad n=1, \ldots, N, \\
\mathcal{L}_{n, 1} g_{1 *}+\mathcal{L}_{n, 2} g_{2 *}=F_{2}\left(a_{n-N}\right), \quad n=N+1, \ldots, 2 N,
\end{gathered}
$$

where

$$
\begin{gathered}
\mathcal{L}_{n, 1} g_{1 *}=\frac{1}{4 \pi k} \int_{\Gamma} g_{1 *}(\sigma) Q^{-1}(\sigma)\left[L_{11}\left(x\left(a_{n}\right), y_{1}(\sigma), y_{2}(\sigma)\right)\right. \\
\left.-L_{11}\left(x\left(a_{n}\right), y_{1}(\sigma),-y_{2}(\sigma)\right)+H_{1}\left(x\left(a_{n}\right), y(\sigma)\right)\right] d \sigma, \quad n=1, \ldots, N ; \\
\mathcal{L}_{n, 2} g_{2 *}=\frac{1}{4 \pi k} \int_{\Gamma} g_{2 *}(\sigma) Q^{-1}(\sigma)\left[L_{12}\left(x\left(a_{n}\right), y_{1}(\sigma), y_{2}(\sigma)\right)\right. \\
\left.-L_{12}\left(x\left(a_{n}\right), y_{1}(\sigma),-y_{2}(\sigma)\right)-H_{2}\left(x\left(a_{n}\right), y(\sigma)\right)+H_{3}\left(x\left(a_{n}\right), y(\sigma)\right)\right] d \sigma, \quad n=1, \ldots, N ; \\
\mathcal{L}_{n, 1} g_{1 *}=\frac{1}{4 \pi k} \int_{\Gamma} g_{1 *}(\sigma) Q^{-1}(\sigma)\left[L_{21}\left(x\left(a_{n-N}\right), y_{1}(\sigma), y_{2}(\sigma)\right)\right. \\
-L_{21}\left(x\left(a_{n-N}\right), y_{1}(\sigma),-y_{2}(\sigma)\right) \\
\left.+H_{2}\left(x\left(a_{n-N}\right), y(\sigma)\right)+H_{3}\left(x\left(a_{n-N}\right), y(\sigma)\right)\right] d \sigma, \quad n=N+1, \ldots, 2 N \\
\mathcal{L}_{n, 2} g_{2 *}=\frac{1}{4 \pi k} \int_{\Gamma} g_{2 *}(\sigma) Q^{-1}(\sigma)\left[L_{22}\left(x\left(a_{n-N}\right), y_{1}(\sigma), y_{2}(\sigma)\right)\right. \\
\left.-L_{22}\left(x\left(a_{n-N}\right), y_{1}(\sigma),-y_{2}(\sigma)\right)+H_{1}\left(x\left(a_{n-N}\right), y(\sigma)\right)\right] d \sigma, \quad n=N+1, \ldots, 2 N .
\end{gathered}
$$

When deriving relations (14), functions from (6) are used.

Thus, the system of equations (9), (10) for the functions $g_{1}(s), g_{2}(s)$ is reduced to the system of equations (13)-(14) for the functions $g_{1 *}(s), g_{2 *}(s)$ and $2 N$ constants $G_{0}, \ldots, G_{2 N-1}$.

It follows from the above arguments that any solution of the system (13)-(14) yields the solution of the system (9), (10).

Note that if $g_{1 *}(s), g_{2 *}(s)$ are continuous functions on $\Gamma$ and satisfy equations (13), then they are Hölder continuous on $\Gamma$, owing to Lemma 1. Namely, from Lemma 1 follows

Lemma 2. Let $\Gamma \in C^{2, \lambda}, \lambda \in(0,1]$, and $f_{1}(s), f_{2}(s) \in C^{0, \beta}(\Gamma), \beta=\min \{1 / 2, \lambda\}$. If the functions $g_{1 *}(s), g_{2 *}(s)$ from $C^{0}(\Gamma)$ obey equations (13), then $g_{1 *}(s), g_{2 *}(s) \in C^{0, \beta}(\Gamma)$.

By Lemma 1, condition $f_{1}(s), f_{2}(s) \in C^{0, \beta}(\Gamma)$ holds if conditions (4) hold. Thus, we will look for functions $g_{1 *}(s), g_{2 *}(s)$ in $C^{0}(\Gamma)$ below. If we find such functions satisfying equations (13), then these functions automatically belong to $C^{0, \beta}(\Gamma)$ by Lemma 2 . 
Since $A_{j l}(s, \sigma) \in C^{0}(\Gamma \times \Gamma)$ by Lemma 1, the integral operators from (13):

$$
\left(\mathcal{A}_{j l} g_{l *}\right)(s)=\int_{\Gamma} A_{j l}(s, \sigma) g_{l *}(\sigma) Q^{-1}(\sigma) d \sigma, \quad j=1,2, \quad l=1,2,
$$

are compact operators in $C^{0}(\Gamma)$. This can be verified directly using the Arzela-Ascoli theorem.

Let us introduce the column $\mathbf{G}=\left(G_{0}, \ldots, G_{2 N-1}\right)^{T}$, consisting of $2 N$ constants. We will consider column $\mathbf{G}$ as an element of the finite-dimensional space $E_{2 N}$. We rewrite equations (13a) and (13b) in the operator form

$$
\begin{aligned}
& g_{1 *}-\mathcal{A}_{11} g_{1 *}-\mathcal{A}_{12} g_{2 *}-P_{1} \mathbf{G}=f_{1}, \\
& g_{2 *}-\mathcal{A}_{21} g_{1 *}-\mathcal{A}_{22} g_{2 *}-P_{2} \mathbf{G}=f_{2},
\end{aligned}
$$

where $P_{1}$ is an operator of multiplication of the row containing $2 N$ elements $P_{1}=$ $\left(-s^{0}, \ldots,-s^{N-1}, 0, \ldots, 0\right)$ by the column $\mathbf{G}=\left(G_{0}, \ldots, G_{2 N-1}\right)^{T} ; P_{2}$ is an operator of multiplication of the row containing $2 N$ elements $P_{2}=\left(0, \ldots, 0,-s^{0}, \ldots,-s^{N-1}\right)$ by the column $\mathbf{G}=\left(G_{0}, \ldots, G_{2 N-1}\right)^{T}$. Note that the operators $P_{1}$ and $P_{2}$, acting from $E_{2 N}$ into $C^{0}(\Gamma)$, are finite-dimensional, and thus compact [12, p. 64].

Let $\mathbf{F}=\left(F_{1}\left(a_{1}\right), \ldots, F_{1}\left(a_{N}\right), F_{2}\left(a_{1}\right), \ldots, F_{2}\left(a_{N}\right)\right)^{T}$ be a column containing $2 N$ constants. The column $\mathbf{F}$, as well as the column $\mathbf{G}$, is considered as an element of the space $E_{2 N}$ of the dimension $2 N$. By $I_{2 N}$ we denote the unit matrix of dimension $(2 N) \times(2 N)$. The matrix $I_{2 N}$ is the unit operator in the space $E_{2 N}$. We rewrite equations (14) in the form of one vector equation

$$
\mathbf{G}-\mathbf{L}_{1} g_{1 *}-\mathbf{L}_{2} g_{2 *}-I_{2 N} \mathbf{G}=\mathbf{F} .
$$

The operator $I_{2 N}$ is a compact operator, since any operator acting in finite-dimensional space is compact [11, p. 222]. Operators $\mathbf{L}_{1}$ and $\mathbf{L}_{2}$ are represented as columns of $2 \mathrm{~N}$ linear continuous functionals:

$$
\mathbf{L}_{l}=\left(-\mathcal{L}_{1, l}, \ldots,-\mathcal{L}_{2 N, l}\right)^{T}, \quad l=1,2 .
$$

Each functional acts from $C^{0}(\Gamma)$ into $E_{1}$, so that

$$
\mathbf{L}_{l} g_{l *}=\left(-\mathcal{L}_{1, l} g_{l *}, \ldots,-\mathcal{L}_{2 N, l} g_{l *}\right)^{T}, \quad l=1,2,
$$

where functionals $\mathcal{L}_{n, l} g_{l *}$ are introduced in (15) for $l=1,2$ and $n=1, \ldots, 2 N$. The operator $\mathbf{L}_{l}$ with $l=1$ or $l=2$, acting from $C^{0}(\Gamma)$ into $E_{2 N}$, is finite-dimensional, and thus compact [12, p. 64].

The first $N$ rows in (17) are equations (14a), while next $N$ rows are equations (14b).

Consider columns $\mathbf{g}=\left(g_{1 *}(s), g_{2 *}(s), \mathbf{G}\right)^{T}$ and $\mathbf{f}=\left(f_{1}(s), f_{2}(s), \mathbf{F}\right)^{T}$ in the Banach space $C^{0}(\Gamma) \times C^{0}(\Gamma) \times E_{2 N}$ with the norm

$$
\|\mathbf{g}\|_{C^{0}(\Gamma) \times C^{0}(\Gamma) \times E_{2 N}}=\left\|g_{1 *}\right\|_{C^{0}(\Gamma)}+\left\|g_{2 *}\right\|_{C^{0}(\Gamma)}+\|\mathbf{G}\|_{E_{2 N}} .
$$

Let us write the system (16), (17) in the form of one equation

$$
(I-R) \mathbf{g}=\mathbf{f}, \quad R=\left(\begin{array}{ccc}
\mathcal{A}_{11} & \mathcal{A}_{12} & P_{1} \\
\mathcal{A}_{21} & \mathcal{A}_{22} & P_{2} \\
\mathbf{L}_{1} & \mathbf{L}_{2} & I_{2 N}
\end{array}\right),
$$

where $I$ is the unit operator in the space $C^{0}(\Gamma) \times C^{0}(\Gamma) \times E_{2 N}$. 
It is shown above that all operators forming $R$ are compact, so $R$ is a compact operator acting from $C^{0}(\Gamma) \times C^{0}(\Gamma) \times E_{2 N}$ into $C^{0}(\Gamma) \times C^{0}(\Gamma) \times E_{2 N}$ (i.e. mapping $C^{0}(\Gamma) \times$ $C^{0}(\Gamma) \times E_{2 N}$ into itself). It follows from the compactness of operator $R$ and from results presented in [12, p. 67], [13, p. 472], [14, p. 221] that equation (18) is a Fredholm equation of the second kind and with index zero in the space $C^{0}(\Gamma) \times C^{0}(\Gamma) \times E_{2 N}$. In other words, Fredholm's alternative holds for equation (18).

Let us show that the homogeneous equation (18) has only a trivial solution in the space $C^{0}(\Gamma) \times C^{0}(\Gamma) \times E_{2 N}$; then nonhomogeneous equation (18) is uniquely solvable in this space due to Fredholm's alternative. Let $\mathbf{g}^{0}=\left(g_{1 *}^{0}(s), g_{2 *}^{0}(s), \mathbf{G}^{0}\right)^{T}$, be an arbitrary solution of the homogeneous equation (18) in the space $C^{0}(\Gamma) \times C^{0}(\Gamma) \times E_{2 N}$, where $\mathbf{G}^{0}=\left(G_{0}^{0}, \ldots, G_{2 N-1}^{0}\right)^{T}$. Hence, the functions $g_{1 *}^{0}(\sigma), g_{2 *}^{0}(\sigma) \in C^{0}(\Gamma)$ and the vector $\mathbf{G}^{0}$ convert homogeneous equations (13), (14) into identities.

By Lemma $2, g_{1 *}^{0}(\sigma), g_{2 *}^{0}(\sigma) \in C^{0, \beta}(\Gamma), \beta=\min \{\lambda, 1 / 2\}$. Consequently, functions

$$
g_{1}^{0}(\sigma)=Q^{-1}(\sigma) g_{1 *}^{0}(\sigma) \in C_{1 / 2}^{\beta}(\Gamma), \quad g_{2}^{0}(\sigma)=Q^{-1}(\sigma) g_{2 *}^{0}(\sigma) \in C_{1 / 2}^{\beta}(\Gamma)
$$

and the vector $\mathbf{G}^{0}$ convert homogeneous equations (12), (10) into identities. In particular, the functions $g_{1}^{0}(\sigma), g_{2}^{0}(\sigma)$ convert homogeneous equations (10) into identities.

Applying the singular integral operator with the kernel $(s-\sigma)^{-1}$ to (12a) and (12b), we obtain that the functions $g_{1}^{0}(\sigma)$ and $g_{2}^{0}(\sigma)$ satisfy homogeneous singular integral equations (11), which are equivalent to homogeneous equations (9). Therefore, the functions $g_{1}^{0}(\sigma), g_{2}^{0}(\sigma) \in C_{1 / 2}^{\beta}(\Gamma)$ satisfy homogeneous equations (9), (10). By Theorem 3, $\left\{v_{1}\left[g_{1}^{0}, g_{2}^{0}\right](x), \quad v_{2}\left[g_{1}^{0}, g_{2}^{0}\right](x), p\left[g_{1}^{0}, g_{2}^{0}\right](x)\right\}$ is a solution of the homogeneous problem $\mathcal{U}$.

By Theorem 1, the homogeneous problem $\mathcal{U}$ has only a trivial solution, i.e.

$$
v_{1}\left[g_{1}^{0}, g_{2}^{0}\right](x) \equiv 0, \quad v_{2}\left[g_{1}^{0}, g_{2}^{0}\right](x) \equiv 0, \quad p\left[g_{1}^{0}, g_{2}^{0}\right](x) \equiv 0, \quad x \in R_{+}^{2} \backslash \Gamma .
$$

Using limiting formulae for the pressure potential [7, 9, on $\Gamma^{+}$and $\Gamma^{-}$, and using limiting formulae from Theorem 2 for normal derivatives of velocity potentials on $\Gamma^{+}$and $\Gamma^{-}$, we obtain

$$
\begin{gathered}
k\left(\left.\frac{\partial v_{j}\left[g_{1}^{0}, g_{2}^{0}\right](x)}{\partial \mathbf{n}_{x}}\right|_{x(s) \in \Gamma^{+}}-\left.\frac{\partial v_{j}\left[g_{1}^{0}, g_{2}^{0}\right](x)}{\partial \mathbf{n}_{x}}\right|_{x(s) \in \Gamma^{-}}\right) \\
-\left(\left.p\left[g_{1}^{0}, g_{2}^{0}\right](x)\right|_{x(s) \in \Gamma^{+}}-\left.p\left[g_{1}^{0}, g_{2}^{0}\right]\right|_{x(s) \in \Gamma^{-}}\right) \cos \left(\mathbf{n}_{x}, x_{j}\right)=g_{j}^{0}(s), \quad s \in \Gamma, \quad j=1,2 .
\end{gathered}
$$

When deriving (20) we used the fact that derivatives in $x_{1}, x_{2}$ of functions $L_{j l}\left(x, y_{1},-y_{2}\right)$ and $H_{m}(x, y)$ from (5) are continuous as $x \rightarrow y \in \Gamma$ for $j, l=1,2$ and $m=1,2,3$. In addition, we took into account that functions

$$
\frac{\cos \left(\psi\left(x, y_{1}(\sigma),-y_{2}(\sigma)\right)\right)}{r\left(x, y_{1}(\sigma),-y_{2}(\sigma)\right)}, \quad \frac{\sin \left(\psi\left(x, y_{1}(\sigma),-y_{2}(\sigma)\right)\right)}{r\left(x, y_{1}(\sigma),-y_{2}(\sigma)\right)}, \quad \frac{H_{1}(x, y(\sigma))}{x_{2}}, \quad \frac{H_{2}(x, y(\sigma))}{x_{2}}
$$

are continuous as $x \rightarrow y \in \Gamma$.

It follows from $(19),(20)$ that $g_{1}^{0}(s) \equiv 0, g_{2}^{0}(s) \equiv 0$ for $s \in \Gamma$, so $g_{j *}^{0}(s)=g_{j}^{0}(s) Q(s) \equiv 0$ for $j=1,2$ and for $s \in \Gamma$.

Since the functions $g_{1 *}^{0}(s), \quad g_{2 *}^{0}(s)$ and the vector $\mathbf{G}^{0}=\left(G_{0}^{0}, \ldots, G_{2 N-1}^{0}\right)^{T}$ satisfy the homogeneous equations (13) and convert them into identities, we observe that these 
identities take the form

$$
\sum_{n=0}^{N-1} G_{n}^{0} s^{n} \equiv 0, \quad \sum_{n=0}^{N-1} G_{n+N}^{0} s^{n} \equiv 0, \quad s \in \Gamma .
$$

It follows from the main theorem of algebra on a number of roots of a polynomial that all coefficients of both polynomials are equal to zero, i.e. $G_{n}^{0}=0$ for $n=0, \ldots, 2 N-1$.

Consequently, all elements of the vector $\mathbf{g}^{0}$ equal zero; i.e. $\mathbf{g}^{0}$ is a trivial solution of the homogeneous equations (18). So we proved that the homogeneous equation (18) has only the trivial solution in the space $C^{0}(\Gamma) \times C^{0}(\Gamma) \times E_{2 N}$. As shown above, equation (18) is a Fredholm equation of the second kind and with index zero in this space. By Fredholm's alternative, the non-homogeneous equation (18) is uniquely solvable in this space [12, p. 67], [13, p. 472], [14, p. 221]. From the above arguments follows

TheOREM 4. If $\Gamma \in C^{2, \lambda}, \lambda \in(0,1]$, then (18) is a Fredholm equation of the second kind and with index zero in the space $C^{0}(\Gamma) \times C^{0}(\Gamma) \times E_{2 N}$. Moreover, equation (18) has a unique solution $\mathbf{g}=\left(g_{1 *}(s), g_{2 *}(s), \mathbf{G}\right)^{T} \in C^{0}(\Gamma) \times C^{0}(\Gamma) \times E_{2 N}$ for any right-hand side $\mathbf{f}=\left(f_{1}(s), f_{2}(s), \mathbf{F}\right)^{T} \in C^{0}(\Gamma) \times C^{0}(\Gamma) \times E_{2 N}$.

From Theorem 4 and Lemma 2 we obtain

Corollary. If $\Gamma \in C^{2, \lambda}, \lambda \in(0,1]$, and if

$$
\mathbf{f}=\left(f_{1}(s), f_{2}(s), \mathbf{F}\right)^{T} \in C^{0, \beta}(\Gamma) \times C^{0, \beta}(\Gamma) \times E_{2 N},
$$

where $\beta=\min \{\lambda, 1 / 2\}$, then the unique solution $\mathbf{g}=\left(g_{1 *}(s), g_{2 *}(s), \mathbf{G}\right)^{T}$ of equation (18) in $C^{0}(\Gamma) \times C^{0}(\Gamma) \times E_{2 N}$ ensured by Theorem 4 belongs to the space $C^{0, \beta}(\Gamma) \times$ $C^{0, \beta}(\Gamma) \times E_{2 N}$.

Let us turn to the analysis of the solvability of system (9), (10). Let conditions (4) hold. Then by Lemma 1 , the functions $f_{1}(s), f_{2}(s)$ belong to the class $C^{0, \beta}(\Gamma)$ with $\beta=\min \{1 / 2, \lambda\}$. According to the corollary to Theorem 4, equation (18) with such functions $f_{1}(s), f_{2}(s)$ has the unique solution $\mathbf{g}=\left(g_{1 *}(s), g_{2 *}(s), \mathbf{G}\right)^{T}$ in the space $C^{0}(\Gamma) \times C^{0}(\Gamma) \times E_{2 N}$, and this solution automatically belongs to $C^{0, \beta}(\Gamma) \times C^{0, \beta}(\Gamma) \times E_{2 N}$. It follows from derivation of equation (18) that the functions

$$
g_{1}(\sigma)=Q^{-1}(\sigma) g_{1 *}(\sigma) \in C_{1 / 2}^{\beta}(\Gamma), \quad g_{2}(\sigma)=Q^{-1}(\sigma) g_{2 *}(\sigma) \in C_{1 / 2}^{\beta}(\Gamma)
$$

and the vector $\mathbf{G}$ convert equations (12), (10) into identities. In particular, functions $g_{1}(\sigma), g_{2}(\sigma)$ convert equations (10) into identities. Applying a singular integral operator with the kernel $(s-\sigma)^{-1}$ to identities $(12 \mathrm{a})$ and $(12 \mathrm{~b})$, we obtain that the functions $g_{1}(\sigma)$ and $g_{2}(\sigma)$ satisfy singular integral equations (11), which coincide with equations (9). Thus, we have proved

TheOREM 5. Let $\Gamma \in C^{2, \lambda} ; F_{1}(s), F_{2}(s) \in C^{1, \lambda}(\Gamma) ; \lambda \in(0,1]$. Then the system of equations (9), (10) has a solution $\left\{g_{1}(s), g_{2}(s)\right\}$ such that $g_{1}(s), g_{2}(s) \in C_{1 / 2}^{\beta}(\Gamma)$ with $\beta=\min \{\lambda, 1 / 2\}$. The functions $g_{1}(s), g_{2}(s)$ in this solution are given by formula $g_{j}(s)=g_{j *}(s) Q^{-1}(s), \quad j=1,2$, where the functions $g_{1 *}(s), g_{2 *}(s) \in C^{0, \beta}(\Gamma)$ are elements of the unique solution $\mathbf{g}=\left(g_{1 *}(s), g_{2 *}(s), \mathbf{G}\right)^{T}=\left(g_{1 *}(s), g_{2 *}(s), G_{0}, \ldots, G_{2 N-1}\right)^{T}$ of equation (18) in the space $C^{0}(\Gamma) \times C^{0}(\Gamma) \times E_{2 N}$, existence of which is ensured by Theorem 4. 
REMARK. Using the technique of the proof of Theorem 4, one can show that the system (9), (10) has no more than one solution $\left\{g_{1}(s), g_{2}(s)\right\}$ such that $g_{1}(s), g_{2}(s) \in C_{q}^{\omega}(\Gamma)$ with $\omega \in(0,1]$ and $q \in[0,1)$. So, the solution ensured by Theorem 5 is unique.

The existence theorem for problem $\mathcal{U}$ follows from Theorem 5 and from Theorem 3.

Theorem 6. Let $\Gamma \in C^{2, \lambda}, F_{1}(s), F_{2}(s) \in C^{1, \lambda}(\Gamma), \lambda \in(0,1]$. Then the solution $\left\{v_{1}\left[g_{1}, g_{2}\right](x), v_{2}\left[g_{1}, g_{2}\right](x), p\left[g_{1}, g_{2}\right](x)\right\}$ of problem $\mathcal{U}$ exists and is given by the formulae (6), (7), where $\left\{g_{1}(s), g_{2}(s)\right\}$ is a solution of the system of equations (9), (10), ensured by Theorem 5 .

By Theorem 1, a solution of problem $\mathcal{U}$ obtained in Theorem 6 is unique. It follows from Theorem 2 that if $\left\{v_{1}\left[g_{1}, g_{2}\right](x), v_{2}\left[g_{1}, g_{2}\right](x), p\left[g_{1}, g_{2}\right](x)\right\}$ is a solution of problem $\mathcal{U}$ obtained in Theorem 6 , then the function $p\left[g_{1}, g_{2}\right](x)$ and functions $\frac{\partial v_{l}(x)}{\partial x_{j}}$ with $l, j=1,2$ satisfy condition (1) with $\varepsilon=-1 / 2$. The explicit formulae for singularities of the gradient of the solution at the ends of the open $\operatorname{arcs} \Gamma$ will be presented in the next section.

Theorem 6 ensures existence of a classical solution of problem $\mathcal{U}$ if $\Gamma \in$ $C^{2, \lambda}, F_{1}(s), F_{2}(s) \in C^{1, \lambda}(\Gamma), \lambda \in(0,1]$. The uniqueness of this solution follows from Theorem 1. On the basis of our analysis, we suggest the following scheme for solving problem $\mathcal{U}$. First, we find the unique solution $\mathbf{g}=\left(g_{1 *}(s), g_{2 *}(s), \mathbf{G}\right)^{T}$ of the Fredholm equation (18), in which $\mathbf{G}=\left(G_{0}, \ldots, G_{2 N-1}\right)^{T}$ and $g_{1 *}(s), g_{2 *}(s) \in C^{0}(\Gamma)$. The functions $g_{1 *}(s), g_{2 *}(s)$ in this solution belong to $C^{0, \beta}(\Gamma), \beta=\min \{1 / 2, \lambda\}$ automatically. Second, we construct the solution $g_{1}(s), g_{2}(s)$ of equations $(9),(10)$ from $C_{1 / 2}^{\beta}(\Gamma)$ by the formula $g_{j}(s)=g_{j *}(s) Q^{-1}(s), j=1,2$. Finally, substituting $g_{1}(s), g_{2}(s)$ into (6), (7), we obtain the solution of problem $\mathcal{U}$.

6. Behaviour of derivatives of velocities at the ends of $\Gamma$. Let $x(d)$ be one of the endpoints of the contour $\Gamma: x(d) \in X ; d=a_{n}$ or $d=b_{n}, n=1, \ldots, N$. Let velocities $v_{1}(x)=v_{1}\left[g_{1}, g_{2}\right](x), v_{2}(x)=v_{2}\left[g_{1}, g_{2}\right](x)$ be elements of the solution of problem $\mathcal{U}$ obtained in Theorem 6. Let us study the asymptotic behaviour of the derivatives $\frac{\partial v_{1}(x)}{\partial x_{1}}$, $\frac{\partial v_{1}(x)}{\partial x_{2}}, \frac{\partial v_{2}(x)}{\partial x_{1}}, \frac{\partial v_{2}(x)}{\partial x_{2}}$ in a neighbourhood of the point $x(d)$ slit along $\Gamma$. We introduce the system of polar coordinates $(|x-x(d)| \cos \varphi,|x-x(d)| \sin \varphi)$ in the neighbourhood of the point $x(d)$. We assume that $\varphi \in(\alpha(d), \alpha(d)+2 \pi)$ if $d=a_{n}$ and $\varphi \in(\alpha(d)-$ $\pi, \alpha(d)+\pi)$ if $d=b_{n}$. We remind the reader that $\alpha(s)$ is an angle between the direction of the $O x_{1}$ axis and the tangent vector $\tau_{x}$ to the contour $\Gamma$ in the point $x(s)$. We set $\alpha(d)=\alpha\left(a_{n}+0\right)$ if $d=a_{n}$, and $\alpha(d)=\alpha\left(b_{n}-0\right)$ if $d=b_{n}$. Thus, the angle $\varphi$ varies continuously in the neighbourhood of the point $x(d)$ slit along the contour $\Gamma$. Denote

$$
g_{j}^{d}(s)=g_{j}(s)|s-d|^{1 / 2}=Q_{1 / 2}^{-1}(s) g_{j *}(s)|s-d|^{1 / 2}, \quad j=1,2,
$$


whence for $j=1,2$ we have at the ends of $\Gamma: g_{j}^{d}(d)=g_{j}^{a_{n}}\left(a_{n}\right)=g_{j}^{a_{n}}\left(a_{n}+0\right)$ if $d=a_{n}$ and $g_{j}^{d}(d)=g_{j}^{b_{n}}\left(b_{n}\right)=g_{j}^{b_{n}}\left(b_{n}-0\right)$ if $d=b_{n}$. We set

$$
\begin{array}{ll}
C_{j}(\varphi, d)=\cos \left(\frac{1}{2} \varphi+\frac{1}{2} \alpha(d)\right)+(-1)^{j} \cos \left(\frac{3}{2} \varphi+\frac{3}{2} \alpha(d)\right) \cos (\varphi-\alpha(d)), \quad j=1,2, \\
S_{j}(\varphi, d)=\sin \left(\frac{1}{2} \varphi+\frac{1}{2} \alpha(d)\right)+(-1)^{j} \sin \left(\frac{3}{2} \varphi+\frac{3}{2} \alpha(d)\right) \cos (\varphi-\alpha(d)), \quad j=1,2 .
\end{array}
$$

Analyzing the behaviour of derivatives $\frac{\partial v_{1}(x)}{\partial x_{1}}, \frac{\partial v_{1}(x)}{\partial x_{2}}, \frac{\partial v_{2}(x)}{\partial x_{1}}, \frac{\partial v_{2}(x)}{\partial x_{2}}$ at the ends of the contour $\Gamma$ with the help of asymptotic formulae obtained in [6], [9, we arrive at

TheOREm 7. Let $v_{1}(x), v_{2}(x)$ be components of the solution of problem $\mathcal{U}$ obtained in Theorem 6, and let $x(d)$ be one of the endpoints of the contour $\Gamma$, i.e. $x(d) \in X, d=a_{n}$ or $d=b_{n}, n=1, \ldots, N$. Then the following formulae describing asymptotic behaviour of derivatives $\frac{\partial v_{1}(x)}{\partial x_{1}}, \frac{\partial v_{1}(x)}{\partial x_{2}}, \frac{\partial v_{2}(x)}{\partial x_{1}}, \frac{\partial v_{2}(x)}{\partial x_{2}}$ as $x \rightarrow x(d)$ hold for points $x$ lying in the neighbourhood of the point $x(d)$ outside $\Gamma$.

If $d=a_{n}$, then

$$
\begin{aligned}
& \frac{\partial v_{1}(x)}{\partial x_{1}}=\frac{1}{4 k\left|x-x\left(a_{n}\right)\right|^{1 / 2}}\left\{g_{1}^{a_{n}}\left(a_{n}\right)\left[\sin \left(\frac{1}{2} \varphi+\frac{1}{2} \alpha\left(a_{n}\right)\right)-\frac{1}{2} S_{1}\left(\varphi, a_{n}\right)\right]\right. \\
& \left.+\frac{1}{2} g_{2}^{a_{n}}\left(a_{n}\right) C_{1}\left(\varphi, a_{n}\right)\right\}+\Omega_{11}\left(x, a_{n}\right), \\
& \frac{\partial v_{2}(x)}{\partial x_{1}}=\frac{1}{4 k\left|x-x\left(a_{n}\right)\right|^{1 / 2}}\left\{g_{2}^{a_{n}}\left(a_{n}\right)\left[\sin \left(\frac{1}{2} \varphi+\frac{1}{2} \alpha\left(a_{n}\right)\right)+\frac{1}{2} S_{1}\left(\varphi, a_{n}\right)\right]\right. \\
& \left.+\frac{1}{2} g_{1}^{a_{n}}\left(a_{n}\right) C_{1}\left(\varphi, a_{n}\right)\right\}+\Omega_{21}\left(x, a_{n}\right), \\
& \frac{\partial v_{1}(x)}{\partial x_{2}}=\frac{1}{4 k\left|x-x\left(a_{n}\right)\right|^{1 / 2}}\left\{g_{1}^{a_{n}}\left(a_{n}\right)\left[-\cos \left(\frac{1}{2} \varphi+\frac{1}{2} \alpha\left(a_{n}\right)\right)-\frac{1}{2} C_{2}\left(\varphi, a_{n}\right)\right]\right. \\
& \left.-\frac{1}{2} g_{2}^{a_{n}}\left(a_{n}\right) S_{2}\left(\varphi, a_{n}\right)\right\}+\Omega_{12}\left(x, a_{n}\right), \\
& \frac{\partial v_{2}(x)}{\partial x_{2}}=\frac{1}{4 k\left|x-x\left(a_{n}\right)\right|^{1 / 2}}\left\{g_{2}^{a_{n}}\left(a_{n}\right)\left[-\cos \left(\frac{1}{2} \varphi+\frac{1}{2} \alpha\left(a_{n}\right)\right)+\frac{1}{2} C_{2}\left(\varphi, a_{n}\right)\right]\right. \\
& \left.-\frac{1}{2} g_{1}^{a_{n}}\left(a_{n}\right) S_{2}\left(\varphi, a_{n}\right)\right\}+\Omega_{22}\left(x, a_{n}\right) .
\end{aligned}
$$


If $d=b_{n}$, then

$$
\begin{aligned}
& \frac{\partial v_{1}(x)}{\partial x_{1}}=\frac{1}{4 k\left|x-x\left(a_{n}\right)\right|^{1 / 2}}\left\{g_{1}^{b_{n}}\left(b_{n}\right)\left[-\cos \left(\frac{1}{2} \varphi+\frac{1}{2} \alpha\left(b_{n}\right)\right)+\frac{1}{2} C_{1}\left(\varphi, b_{n}\right)\right]\right. \\
& \left.+\frac{1}{2} g_{2}^{b_{n}}\left(b_{n}\right) S_{1}\left(\varphi, b_{n}\right)\right\}+\Omega_{11}\left(x, b_{n}\right) \\
& \frac{\partial v_{2}(x)}{\partial x_{1}}=\frac{1}{4 k\left|x-x\left(a_{n}\right)\right|^{1 / 2}}\left\{g_{2}^{b_{n}}\left(b_{n}\right)\left[-\cos \left(\frac{1}{2} \varphi+\frac{1}{2} \alpha\left(b_{n}\right)\right)-\frac{1}{2} C_{1}\left(\varphi, b_{n}\right)\right]\right. \\
& \left.+\frac{1}{2} g_{1}^{b_{n}}\left(b_{n}\right) S_{1}\left(\varphi, b_{n}\right)\right\}+\Omega_{21}\left(x, b_{n}\right) \\
& \frac{\partial v_{1}(x)}{\partial x_{2}}=\frac{1}{4 k\left|x-x\left(a_{n}\right)\right|^{1 / 2}}\left\{g_{1}^{b_{n}}\left(b_{n}\right)\left[-\sin \left(\frac{1}{2} \varphi+\frac{1}{2} \alpha\left(b_{n}\right)\right)-\frac{1}{2} S_{2}\left(\varphi, b_{n}\right)\right]\right. \\
& \left.+\frac{1}{2} g_{2}^{b_{n}}\left(b_{n}\right) C_{2}\left(\varphi, b_{n}\right)\right\}+\Omega_{12}\left(x, b_{n}\right) \\
& \frac{\partial v_{2}(x)}{\partial x_{2}}=\frac{1}{4 k\left|x-x\left(a_{n}\right)\right|^{1 / 2}}\left\{g_{2}^{b_{n}}\left(b_{n}\right)\left[-\sin \left(\frac{1}{2} \varphi+\frac{1}{2} \alpha\left(b_{n}\right)\right)+\frac{1}{2} S_{2}\left(\varphi, b_{n}\right)\right]\right. \\
& \left.+\frac{1}{2} g_{1}^{b_{n}}\left(b_{n}\right) C_{2}\left(\varphi, b_{n}\right)\right\}+\Omega_{22}\left(x, b_{n}\right) .
\end{aligned}
$$

By $\Omega_{l j}(x, d)$ with $l, j=1,2$, we denote functions that are continuous in $x$ in the neighbourhood of the point $x(d)$ slit along $\Gamma$ and are continuously extensible onto $\Gamma \backslash x(d)$ from the left and from the right from this neighbourhood, but these functions may be unbounded in the point $x(d)$ and satisfy the following inequality:

$$
\left|\Omega_{l j}(x, d)\right| \leq \frac{\text { const }}{|x-x(d)|^{q_{1}}}, \quad l, j=1,2
$$

for all points $x$ lying in the neighbourhood of the point $x(d)$ outside $\Gamma$. Here const is some constant and $q_{1}$ is any number such that $q_{1} \in(1 / 2-\beta, 1 / 2), \beta=\min \{1 / 2, \lambda\}$.

It follows from Theorem 7 that the derivatives $\frac{\partial v_{l}(x)}{\partial x_{j}}, l, j=1,2$ behave near the end $x(d)$ of the contour $\Gamma$ as $O\left(|x-x(d)|^{-1 / 2}\right)$ in the general case. However, if $g_{1}^{d}(d)=$ $g_{2}^{d}(d)=0$, then these derivatives have less singularity in the point $x(d)$, since they satisfy the inequality

$$
\left|\frac{\partial v_{l}(x)}{\partial x_{j}}\right| \leq \frac{\text { const }}{|x-x(d)|^{q_{1}}}, \quad l, j=1,2
$$

for any $q_{1}$ pointed out in Theorem 7 , i.e. for any $q_{1} \in(1 / 2-\beta, 1 / 2)$. Inequality (22) holds for any $x$ lying near $x(d)$ outside $\Gamma$. Note that if $\lambda \in[1 / 2,1]$ in Theorem 6 , then $\beta=1 / 2$ and inequalities (21), (22) hold for any $q_{1} \in(0,1 / 2)$.

The research was partially supported by the RFBR grants No. 08-01-00082 and 10-01-00009.

\section{REFERENCES}

[1] Popov A.N. Application of potential theory to solving the linearized Navier-Stokes system of equations in a two-dimensional case. Trudy MIAN, 1971, t. 116, pp. 162-180. (In Russian). MR0364909 (51:1163) 
[2] Pozrikidis C. Boundary integral and singularity method for linearized viscous flow. Cambridge University Press, Cambridge, 1992. MR,1156495 (93a:76027)

[3] Varnhorn W. The Stokes equations. Akademie Verlag, Berlin, 1994. MR.1282728 (95e:35162)

[4] Power H. The completed double layer boundary integral equation method for two-dimensional Stokes flow. IMA Journal of Applied Mathematics, 1993, v. 51, pp. 123-145. MR1244192 (94i:76020)

[5] Vladimirov V.S. Equations of Mathematical Physics. Nauka, Moscow, 1981. (In Russian; English translation of 1st edition: Marcel Dekker, N.Y., 1971.)

[6] Krutitskii P.A. Dirichlet problem for the Helmholtz equation outside cuts in a plane. Comp. Maths. Math. Phys., 1994, v. 34, No. 8/9, pp. 1073-1090. MR1300397 (95f:35046)

[7] Krutitskii P.A. On Stokes potential for pressure. Russian mathematical surveys, 2007, v. 62, No. 2, pp. 385-387. MR2352372

[8] Krutitskii P.A. On Stokes potentials for velocities. Russian mathematical surveys, 2007, v. 62, No. 6, pp. 1212-1214. MR2382807 (2008k:35378)

[9] Krutitskii P.A. On properties of some integrals related to potentials for Stokes equations. Quarterly of Applied Mathematics, 2007, v. 65, No. 3, pp. 549-569. MR2354887 (2008i:31003)

[10] Muskhelishvili N.I. Singular integral equations. Nauka, Moscow, 1968. (In Russian; English translation: Noordhoff, Groningen, 1972.) MR0355494(50:7968)

[11] Kolmogorov A.N., Fomin S.V. Elements of function theory and functional analysis. Nauka, Moscow, 1981 (in Russian). English translation: Dover, New York, 1999. MR630899 (83a:46001)

[12] Krein S.G. (editor). Functional analysis. Nauka, Moscow, 1964 (in Russian). English translation: Wolters-Noordhoff Publishing, Groningen, 1972.

[13] Kantorovich L.V., Akilov G.P. Functional Analysis. Nauka, Moscow, 1984 (in Russian). English translation: Pergamon Press, Oxford, 1982. MR664597 (83h:46002)

[14] Trenogin V.A. Functional Analysis. Nauka, Moscow, 1980 (in Russian). French translation: Mir Publishers, Moscow, 1985. MR836334 (87g:46001)

[15] Krutitskii P.A. An explicit solution of the pseudo-hyperbolic initial boundary value problem in a multiply connected region. Mathematical methods in the applied sciences, 1995, v. 18, pp. 897-925. MR1346665 (97d:76010a)

[16] Bruno A.D. Power Geometry in Algebraic and Differential Equations. Elsevier, Amsterdam, 2000. MR 1773512 (2002c:37071)

[17] Kohr M. Existence and uniqueness result for Stokes flow in a half-plane. Arch. Mech., 1998, v. 50, No. 4, pp. 791-803. MR.1670707 (99m:76049)

[18] Power H., Power B.F. Second kind integral equation formulation for the slow motion of a particle of arbitrary shape near a plane wall in a viscous fluid. SIAM J. Appl. Math., 1993, v. 53, No. 1, pp. 60-70. MR1202840 (93i:76033)

[19] Hsu R., Canatos P. The motion of a rigid body in a viscous fluid bounded by a plane wall. J. Fluid Mech., 1989, v. 207, pp. 29-72. 